\title{
Experiences when irradiating grafts and flaps for skin
} cancer

\begin{abstract}
Resection of skin cancer with graft or flap repair may be referred for local postoperative radiotherapy (PORT) to ensure local control. PORT needs to be delivered in a certain time frame after surgery, but this may impact on graft and flap survival. The purpose of this article is to pass on some experience our multidisciplinary team has acquired in order to assist in the management of these cases. Specifically, pertinent issues in patient assessment (history and examination), indications for PORT, the differences between a graft and a flap, and technical considerations in the prescription of radiotherapy to these are covered, accompanied by illustrative cases with appropriate photographs and diagrams. The principal findings are that a conservative approach may need to be taken to ensure graft and flap survival, with PORT reserved for a role in salvage. Other interesting cases cover the lack of acute radiation side effects in flaps; the tendency of acute radiation skin toxicity to move with gravity, and transplanted skin may have a different radiation sensitivity to that of its new environment. We hope that this article will be of use for the multidisciplinary skin oncology team. Further research is needed to validate and confirm our findings.
\end{abstract}

Volume 7 Issue 3 - 2020

\author{
Liam J Dwyer,' Roger L Haddad, ${ }^{2}$ Gerald B \\ Fogarty \\ 'St Vincent's Clinical School, Faculty of Medicine, UNSW, \\ Australia \\ ${ }^{2}$ Department of Plastic Surgery, St Vincent's Hospital,Australia \\ ${ }^{3}$ GenesisCare, Department of Radiation Oncology, St Vincent's \\ Hospital,Australia
}

\begin{abstract}
Correspondence: Gerald B Fogarty, GenesisCare, Department of Radiation Oncology, St Vincent's Hospital, 390Victoria Street, Darlinghurst NSW 2010 Australia,
\end{abstract}

Email Gerald.fogarty@genesiscare.com

Received: May 14, 2020 | Published: May 25, 2020

Keywords: skin neoplasms, surgical flaps, skin transplantation, radiotherapy, adjuvant, radiation tolerance, patient care team

Abbreviations: PORT, postoperative radiotherapy; LC, local control; PNI, perineural invasion; LVSI, lymphovascular invasion; Gy, gray; RT, radiotherapy; UV, ultraviolet; $\mathrm{BCC}$, basal cell carcinoma; cSCC, cutaneous squamous cell carcinoma; RCTs, Randomised controlled trials; RO, Radiation Oncologist; CTV, clinical target volume; PTV, planning target volume; SXRT, Superficial X-ray Radiation Therapy; CT, computed tomograph; $\mathrm{cm}$, centimetre; $\mathrm{mm}$, millimetre; STSG, split-thickness skin graft; FTSG, full-thickness skin graft; RCM, reflectance confocal microscopy

\section{Introduction}

Postoperative Radiotherapy (PORT) following resection of skin cancer is a common alternative to more surgery when a histopathology report unexpectedly reveals locally advanced disease needing further treatment to ensure local control (LC). Examples of locally advanced disease include extra-tumoral perineural invasion (PNI), lymphovascular invasion (LVSI), positive margins and/or invasion of other structures. ${ }^{1-5}$ The main advantage of PORT is there is no further tissue sacrifice which may compromise function and cosmesis in sensitive areas e.g., nose, ears. PORT needs to be given within a certain time frame following surgery before any macroscopic recurrence can develop. PORT often needs to be delivered to the surgical bed which has been closed using a graft or flap but then there is a risk that PORT may cause graft or flap failure. This article will address some issues around this problem based on experiences with individual cases.

\section{Assessing the patient}

\section{History}

Attaining specific histories of patient, tumour and its treatment are fundamental in this scenario, especially details that may lead to decreasing the overall radiation dose which will decrease the chance of graft or flap failure. Specific history of patient factors includes history and duration of any immunosuppression eg history of transplant and current levels of immunosuppressive medicines, chronic lymphocytic leukaemia, human immunodeficiency virus infection and treatment. Often these patients are more radiosensitive for reasons yet to be elucidated. ${ }^{6}$ Standard fractionation of 2 gray (Gy) per day or less means that the acute radiation side effects occurring at any given time in treatment corresponds to the dose delivered at that stage. ${ }^{7}$ Hypofractionated courses, common in skin cancer, often have a component of acceleration and toxicity can peak after the dose is given. This means titration to the acute reaction of the day cannot be done. Standard fractionation and regular physician review enables dose titration, and a lower overall dose may be all that is needed.

Previous skin cancers and their treatment and outcomes may lead to finessing the radiation dose needed. Previous radiation to the index site will affect the radiation script, often leading to more hyperfractionation. Drug history needs to include drugs that are radiation sensitising eg methotrexate, hydroxyurea. If these are necessary to take during radiation, a close eye on acute toxicity is needed as the dose may need to be reduced, given the sensitising effect. Standard fractionation of $2 \mathrm{~Gy}$ or lower per day is also advised. There may be a history of skin cancer being caused by an agent other than ultraviolet (UV) light and this may influence total radiotherapy (RT) dose. UV induced lesions also tend to be more radiation sensitive, also for reasons yet to be elucidated.

Past and family history of skin cancer, other cancers, and unusual toxicities from previous radiation treatments may lead to a clinical suspicion of a rare skin cancer syndrome (eg Gorlin syndrome) or radiation sensitivity syndrome (e.g., mosaics of xerodermapigmentosum $)^{8}$ and may prompt a genetic review. Support that the patient has, especially for transport to and from a fractionated treatment, help with dressings and with the activities of daily living, including food preparation, are important to ascertain prior to PORT. Food preparation is important as good nutrition is needed to ensure repair 
of normal tissues during and after PORT, otherwise acute reactions can be more severe, leading to inability to complete the RT script. Problems in these areas may even push the team to consider another therapy, for example, more surgery rather than PORT to guarantee LC. A specific history of tumour factors needs to include time from when the cancer started, and any symptoms including bleeding, ulceration, the need for dressings, a lesion that crusts then resolves and crusts again, so typical of basal cell carcinoma (BCC). This will give a clue as to when recurrence can be expected and can help the RO make sure PORT begins promptly. Perineural symptoms such as pain, numbness and formication on or around the lesion need to be asked about, as this can change the PORT volume to be treated. Lymph node symptoms need to be addressed, eg neck and groin swellings, as the patient or even previous medical attendants may not associate these with the primary disease or even be aware of them nor do an examination for them. A specific history of previous treatment needs to be elicited, especially previous surgery for cutaneous squamous cell carcinoma $(\mathrm{cSCC})$ as recurrence in this scenario is an absolute indication on the EViQ website for PORT. ${ }^{2}$

\section{Examination}

Examination particularly involves inspection and palpation. Persistent or even already recurrent disease may be seen at the edge of a graft where there was a positive margin. Experience, repeat biopsy and often clinical involvement of the surgeon, is needed to ensure this is only granulation tissue. Macroscopic recurrence may be felt under a flap where there was a positive deep margin, often depending on duration since surgery. Draining lymph node stations need to be palpated especially the epitrochlear node in the upper limb and popliteal node station in the lower, often missed by physicians who are not mainstream skin cancer surgeons. Pre-existing graft and flap failure needs to be assessed and documented. Failure is problematic. Studies show that PORT needs to start within a certain timeframe, otherwise LC and even survival can be compromised. ${ }^{9}$ Failure can often mean re-operation or healing by secondary intention which can further delay or even invalidate PORT. Multidisciplinary discussion with the surgical team is necessary in this scenario. The histopathology report needs to be interrogated to adequately stage the cancer. Details of locally advanced disease include PNI, LVSI, positive margins and or invasion of other structures. Unfortunately the data on when PORT should be given is not of robust quality , $3,10-12$ and often a guess for what is the risk of recurrence without PORT has to be made, based on the relative number of favourable and unfavourable prognostic factors discovered in the patient assessment. These are listed in Table 1. Randomised controlled trials (RCTs) are needed.

Table I Factors influencing the decision to prescribe local RT

\begin{tabular}{lll}
\hline Factor & $\begin{array}{l}\text { Presence of this } \\
\text { factor favours: }\end{array}$ \\
\hline Patient & $\begin{array}{l}\text { Immunosuppression } \\
\text { Challenged mobility/ } \\
\text { home support to attend } \\
\text { fractionated therapy }\end{array}$ & Rx other than RT \\
& $\begin{array}{l}\text { Low need for function/ } \\
\text { cosmesis } \\
\text { Radiation sensitivity } \\
\text { syndrome/drugs }\end{array}$ & Rx other than RT \\
\hline
\end{tabular}

Table Continued...

\begin{tabular}{lll}
\hline Factor & & $\begin{array}{l}\text { Presence of this } \\
\text { factor favours: }\end{array}$ \\
\hline Tumour & $\begin{array}{l}\text { Recurrent Disease } \\
\text { PNI, LVSI especially if extra } \\
\text { tumoral } \\
\text { Close/Positive margin }\end{array}$ & RT \\
& High risk site - eg ear & RT \\
& Depth over 4mm & $\mathrm{RT}^{13}$ \\
Treatment & $\begin{array}{l}\text { Previous RT to index site } \\
\text { Graft/flap at risk of failure if }\end{array}$ & Rx other than RT \\
& RORT added & other than RT \\
\hline
\end{tabular}

\section{PORT after simple closure}

PORT following simple excision and closure is straightforward. Clinical macroscopic recurrence often found by palpation for a mass deep to the scar will increase the total dose needed. At planning, the Radiation Oncologist (RO) draws a line on the scar. The tumour is assumed to be in the middle of the scar. The scar is assumed to be the centre of the clinical target volume (CTV). ${ }^{15}$ There is little data about how far the CTV should extend beyond the scar but most would give a 1-2 centimetre $(\mathrm{cm})$ margin on the scar to CTV. This is extended by a planning target volume (PTV) ${ }^{15}$ which partly depends on what modality is used. A I cm margin from CTV to PTV is given for megavoltage electrons, and usually narrower expansion for superficial (SXRT) or orthovoltage photon RT. Often in an experienced unit the only mark given by the RO is the field. These marks need to be stored using a planning template, so that future true local recurrence can be differentiated from regional recurrence. Photos are taken of the set up to aid with treatment positioning. If computer planning is being used, these marks are overlaid by wire to capture them on the planning computed tomograph (CT). Computer contouring assists in getting the treatment depth correct which should be gleaned from a complete histopathology report. This is particularly important for ensuring adequate dose to a positive deep margin.

\section{About grafts and flaps}

A skin cancer is removed by local excision which includes a margin. Clinical margins at the time of surgery are a balance between clearance and tissue conservation. A well demarcated tumour in a cosmetically sensitive area, such as a nodular BCC in the nose can often be safely removed with a 2-3 millimetre $(\mathrm{mm})$ margin of normal tissue. As the tumour becomes larger, more aggressive, and the edges become less distinct, a wider margin is required to achieve surgical clearance and reduce recurrence. Hence, $5 \mathrm{~mm}$ may be sufficient for a well to moderately differentiated SCC; a poorly differentiated SCC probably requires a $1 \mathrm{~cm}$ margin, again balancing tumour factors with location and patient factors. Clearance by one anatomical plane beyond the tumour is usually considered an adequate deep margin for SCC and BCC. RCTs have shown melanoma needs a skin margin of cms. ${ }^{16,17}$ The currently accruing MelMarT trial is assessing one versus two $\mathrm{cm}$ margins. ${ }^{18}$ The margin is also influenced by the position of the lesion on the body e.g., nose versus back. Deep margin is taken to the deep fascia for melanoma. A skin graft is harvested from a different site on the same patient. It is completely detached from the donor site and transferred to the defect. A split-thickness skin graft (STSG) includes the epidermis and part of the dermis. A full-thickness skin graft (FTSG) consists of the epidermis and the entire thickness of the 
dermis. When more than one tissue type is included in the graft, such as skin and cartilage, this is called a composite graft. Grafts depend on the recipient bed for nutrition. Graft "take" depends on vascularity of the recipient site, and so these generally don't survive well if placed on avascular beds such as exposed bone or tendon.

A skin flap can be of several types. Unlike grafts, flaps retain an intact blood supply to the skin. Local flaps share a common border with the defect and are designed from the adjacent skin. They are elevated and turned into the wound. The movement of a local flap can include advancement, rotation, transposition, or any combination of these. Advancement flaps move skin in line with the flap. A rotation flap is similar except the flap is stretched in an arc. When there is insufficient laxity immediately adjacent to the defect, a transposition flap can be used which involves turning a flap transversely over an intact adjacent piece of tissue. The transposition flap is commonly used on the scalp, and results in a new secondary defect which must then be closed, usually by a graft. Because local flaps bring skin from the adjacent area, they often have the best colour match and generally allow better contouring than a skin graft. Local flaps and full thickness skin grafts are the two workhorse techniques used for repair of most facial skin cancer defects. Regional flaps import tissue from a nearby area, but without sharing a border with the defect. These can include skin, muscle, and several other tissue types depending on location. The regional flap needs to be moved over or under intact skin to reach the defect. Again, they retain a blood supply connected to the donor site via a pedicle, and are often designed around these feeding blood vessels. Over time, a regional flap recruits vascularity from its new location, and becomes less reliant on its feeding pedicle. This however may not be the case for pedicled muscle flaps, or if the flap is placed on a defect with impaired vascularity, such as after previous high dose irradiation. Distant flaps, or free flaps, are when a flap is raised with its pedicle, completely detached from the body, and transferred to the defect. The blood supply is cut and then reattached microsurgically to a new blood supply at the recipient site. These reconstructions are more involved and time consuming but have become the gold standard for many complex skin cancer defects.

\section{PORT after grafts and flaps}

The oncological operation leaves a skin defect. If the defect is not appropriate for direct closure, then either a skin graft or flap is needed for coverage. PORT following grafts and flaps is then more challenging as graft or flap survival needs to be considered in the radiation total dose, fractionation pattern, volume irradiated and how soon post operation the RT should be given. An advantage of free flaps is that the blood supply is immediately established limiting the time needed for vascularistion before starting PORT. Decreasing delay is important as surgery releases growth factors that help the normal tissues to heal. Cancer cells may also respond, and may rapidly grow in the surgical bed as their growth is not controlled by the body's normal homeostatic mechanisms. ${ }^{19}$ PORT dosing schedules assume that there is only microscopic disease present, hence it is important to start RT within a certain time after operation before macroscopic growth has occurred. Macroscopic growth may occur prior to PORT starting if there is excessive delay for healing. Macroscopic growth prior to PORT may require increasing total RT dose. Regrowth may even need a further excision prior to PORT, worsening treatment outcomes in terms of increasing the possibility of regional spread and increasing normal tissue sacrifice which will impact function and cosmesis. PORT needs to be aimed at the lesion bed in a process called planning. Pre-surgery photos and drawings are a great help. RO mantra is that the whole surgical bed is at risk and ROs will try to cover this with a CTV to at least enough doses for microscopic residual disease. In simple closure the surgical bed is usually easy to define and the lesion bed and surgical bed are almost identical (Figure $1)$.

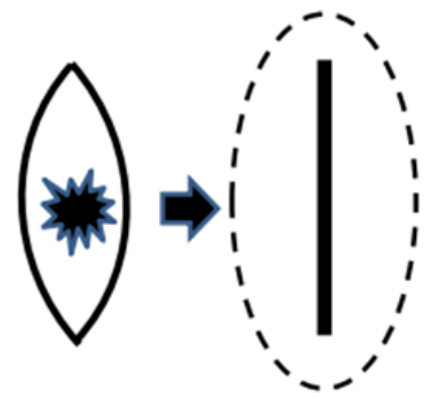

Figure I Simple excision of lesion and closure of wound with straight scar surrounded by radiation field.

When it not possible to approximate the sides of the surgical cavity for whatever reason, a graft or flap must be used. If locally advanced disease is then found on histopathology, a RO referral may be made and it will be up the RO to assess whether RT is the best way of ensuring local control. When a skin graft is used to repair a wider surgical defect, the corresponding RT field is then wider (Figure 2) and the greater volume must be taken into account. This may lead to a change in RT modality. For example, most SXRT units have an effective maximum field diameter of treatment of $8 \mathrm{~cm}$. Increasing the width may lead to having to use electron megavoltage therapy, with its added complication of needing a mask for head and neck cases, and bolus to get a therapeutic surface dose

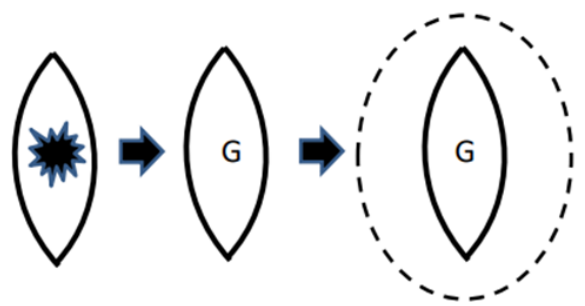

Figure $\mathbf{2}$ Skin tumour removed and surgical defect is covered with a skin graft $(\mathrm{G})$. The surgical bed is wider and the corresponding RT field is wider as well to cover all the surgical bed.

Grafts get their blood supply from the surgical bed and skin margins. When used directly on the skull, large grafts may fail centrally as vessels may be slow to grow to the centre (Figure 3A\&3B). Immediate PORT prior to central vascularisation will cause the graft to fail centrally. More bone will be exposed. In fit patients, the defect may eventually close by secondary intention. In others, the bone remains exposed. Salvage with a flap may be needed. There is no danger in exposed bone, but the patient is often unhappy with the functional and cosmetic result. In the case in Figure 3, another approach would be to wait until the graft has completely taken and is centrally re-perfused. This can be tested clinically by the graft blanching on being pressed, then re-perfusing on release. PORT can then be given more safely. If the wait has been a long time, some may question whether PORT should then be given anyway. If a biopsyproven recurrence occurs in meantime, salvage lumpectomy followed by flap and PORT to the surgical bed can then be done. 




Figure 3 PORT to grafted surgical bed on scalp causes further graft failure. (A) At planning. Patient referred for PORT for positive deep surgical margin of BCC with a large split skin graft covering forehead defect. This graft is already failing. (B) Three months post PORT.The graft failed centrally, and the defect is healing by secondary intention.

A full thickness skin graft is likely to be more resistant to radiotherapy than a split thickness skin graft presumably as its thickness makes it more able to be more quickly vascularised and therefore more robust. A graft placed on a soft tissue base is also likely more radioresistant than a graft placed over a thin layer of periosteum due also presumably to speedier vascularisation. From the above it can be appreciated that ROs favour flaps. Surgeons who perform flap surgery know that the lesion that was removed, and the corresponding defect, are under the apex of the flap. Figure 4 shows this clearly. This man has had a rotation flap to cover a defect following removal of a $13 \mathrm{~mm}$ cSCC with positive deep margin. He had a photo of where the primary was prior to operation. At planning, when asked, he put his finger on the original location of the primary, which is marked with X, directly under the apex of the flap. ROs may not appreciate this. ROs may place the position of the primary in the middle of the surgical bed. In this case, there was no palpable macroscopic residual or recurrent tumour present clinically, and there was no issue of further hair loss, and so the resultant electron field could be generous. However if a boost dose was needed to the lesion bed because of macroscopic recurrence already at planning, or if only the lesion bed was to be treated, and not the whole surgical bed, then the RO could have made a geographic miss.

\section{When immediate PORT is not given due to concerns of graft or flap survival}

This may happen when the multidisciplinary team decide that PORT may negatively impact the graft or flap, and a conservative approach is adopted. This involves close supervision e.g., every 3 months for 2 years and then salvage treatment given if and when a biopsy proven recurrence is detected. This supervision can be shared amongst the team e.g., by both surgeon and RO. Examination for both local and regional recurrence must be done at follow up. Careful explanation to patient and family is needed to ensure compliance with the review schedule. The adoption of this approach depends on the danger of recurrence which is higher for melanoma and $\mathrm{CSCC}$ than BCC. ${ }^{20-22}$ Adoption will also depend on the impact that graft or flap failure will have on function and cosmesis, and the ability to salvage.



Figure 4 This man has had a rotation flap to cover a defect following removal of a $13 \mathrm{~mm}$ cSCC with positive deep margin. Thin long vertical arrow shows where the patient identified at $X$ where the lesion was prior to operation. $X$ is at apex of flap. Thick short horizontal arrows show beginning and end of rotation flap scar. Thick short vertical arrow show RT electron field outline. ROs may not understand this and put the lesion at the centre of the flap, risking geographic miss.

\section{Experiences during RT delivery}

\section{Flaps may not exhibit acute effects}

Flaps may not exhibit the expected acute radiation toxicity in skin despite the correct dose being delivered. See Figure 5\&6. The cause of this is unknown. One hypothesis is that the transplanted skin comes from areas of the body that have had less UV exposure. Another hypothesis is that the flap is hypoxic, and this may decrease the ability of RT to cause inflammation in transplanted tissue. However, usually the tissue shows no other signs of hypoxia. See Figure 6 . The transplanted flap tissue had enough blood in it to support a regrowth of lentigo maligna. For ROs who treat to a certain level of reaction, this phenomenon may move them to consider increasing the dose to achieve the desired skin reaction. This may be a mistake. Fortunately skin RT lends itself to in vivo dosimetry to check that the planned dose is being delivered.

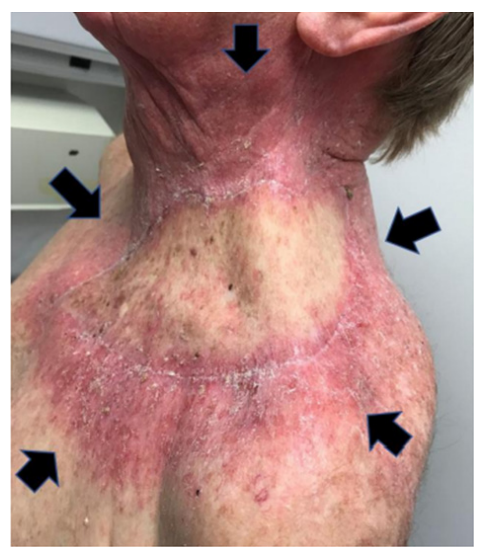

Figure 5 Free flap to trunk. Patient is towards the end of PORT following resection of lesion with high risk features for local and regional recurrence of left supraclavicular fossa. Black arrows show outline of RT field. Notice that the central flap is not as erythematous as the surrounding tissue, despite the same dose being delivered, as checked by in vivo dosimetry. 




Figure 6 Free flap to face. Patient is towards the end of salvage RT following failed free flap repair of defect after resection of lentigo maligna of right face. The flap itself had become involved with biopsy proven recurrence and was being treated to the same dose as the surrounding involved skin. Notice that the flap is not as erythematous as the surrounding tissue despite the same dose being delivered. She was free of disease in the irradiated areas two years after treatment on reflectance confocal microscopy (RCM).

\section{Acute skin toxicity can move according to gravity}

RT causes acute inflammation. ROs are used to seeing this in-field, in fact, even using the reaction to define the field and to help with quality assurance that the planned area is being treated. In those who have had many surgical procedures, RT acute toxicity can start to move with gravity (See Figure 7).



Figure 7 This octogenarian has had significant prior surgical treatment for lentigo maligna. The latest graft was to the left forehead. Unfortunately there were multiple positive margins and there was significant amelanotic areas identified on RCM. He declined further surgery and was treated with salvage RT. The circle is the current RT field. Black arrows show that RT inflammation has tracked down around the orbit to the left cheek but has not gone underneath an older graft on the cheek. There is no RT being delivered to the orbit nor cheek.

\section{Transplanted skin may have different radiation sensitivity to that of its new environment (See Figure 8).}

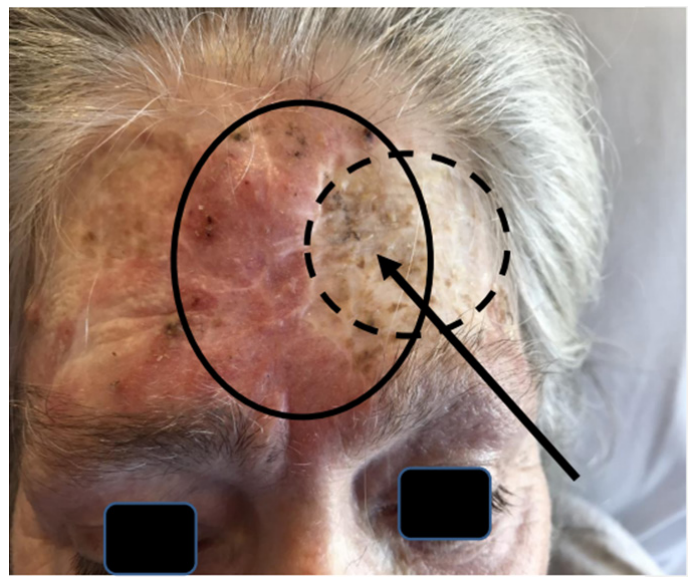

Figure $\mathbf{8}$ This longstanding transplant recipient developed many problems with cSCC. She had had a graft taken from the neck to fill a defect on the left forehead some years previously (see oval with dotted outline).A midline lesion was recently removed but had positive deep margin and needed PORT. The PORT electron field (see oval with solid outline) overlapped onto some of the old graft site. The skin in the old graft site has developed hyperpigmentation, but only that within the current field being irradiated, whereas the rest of the graft area remains the same. The original skin of the area is developing erythema. This erythema is also moving in the direction of gravity like Figure 7 , this time into the bridge of the nose.

\section{Conclusion}

Resection of skin cancer with graft of flap repair may be referred for PORT as an alternative to more surgery when a histopathology report unexpectedly reveals locally advanced disease needing further treatment to ensure LC. PORT needs to be given within a certain time frame following surgery and there is a risk of graft or flap failure. This article is designed to assist ROs and addresses some issues around this problem, and also what may be expected as the therapy is delivered, all based on experiences with individual cases in a multidisciplinary environment involving surgeons and ROs. Issues covered include: issues of importance in patient assessment for defining RT parameters; what is a graft and a flap and the differences, and what the difference between the surgical and lesion bed is, especially for flaps. We also address when PORT may be avoided and a more conservative approach be instituted and the need for multidisciplinary involvement in that decision. Experiences during the therapy such as decreased erythema of the flap, movement of acute RT inflammation with gravity to areas out of field, and transplanted skin maintaining the radio sensitivity of skin of its origin are addressed. Multidisciplinary discussion of cases requiring PORT can help in understanding the reasons for PORT referral and assist in defining the radiation parameters needed for each case. More prospective research is needed to validate and confirm these findings.

\section{Acknowledgments}

We are indebted to the professionalism of the staff of Genesis Care for their commitment to excellence in radiation delivery.

\section{Funding}

None. 


\section{Conflicts of interest}

Author declares that there is no conflict of interest.

\section{References}

1. Seth R, Messersmith H, Kaur V, et al. Systemic therapy for melanoma: ASCO guideline. J Clin Oncol. 2020:JCO-20.

2. EVIQ. Skin cancer non melanoma squamous cell carcinoma high risk adjuvant EBRT post operative. 2015.

3. EVIQ. Skin cancer melanoma adjuvant EBRT post operative. Sydney NSW: EVIQ; 2015.

4. Cancer Council Australia Keratinocyte Cancers Guideline Working Party. Clinical practice guidelines for keratinocyte cancer. Sydney: Cancer Council Australia.

5. Cancer Council Australia Melanoma Guidelines Working Party. Clinical practice guidelines for the diagnosis and management of melanoma. Sydney: Cancer Council Australia.

6. Fogarty GB, Christie D, Potter A. Volumetric modulated arc therapy (VMAT) for extended skin field cancerisation (ESFC): Radiobiological learnings from unique patient cases. Int $J$ Radiol Radiat Ther. 2019;6(5):156-162.

7. Fogarty GB, Christie DRH, Kaminski A, et al. A radiation oncology approach for using definitive radiotherapy with volumetric modulated arc therapy (VMAT) for skin field cancerisation (SFC). Int J Radiol Radiat Ther. 2018;5(4):227-234.

8. Arlett $\mathrm{CF}$, Green $\mathrm{MH}$, Rogers $\mathrm{PB}$, et al. Minimal ionizing radiation sensitivity in a large cohort of xeroderma pigmentosum fibroblasts. $\mathrm{Br} J$ Radiol. 2008;81(961):51-58.

9. Fogarty GB, Burt J, Ainslie J. Delay of post operative radiotherapy in high risk skin cancer can be associated with recurrence. J Plast Reconstr Aes. 2006;59(2):203-205.

10. NCCN. Squamous Cell Skin Cancer. Plymouth Meeting PA: NCCN; 2020.
11. NCCN. Cutaneous Melanoma. Plymouth Meeting PA: NCCN; 2020.

12. NCCN. Basal Cell Carcinoma. Plymouth Meeting PA: NCCN; 2020.

13. Brierley JD, Gospodarowicz MK, Wittekind C, editors. Skin Tumours. TNM Classification of Malignant Tumours (8th edition). Oxford, UK: Wiley-Blackwell; 2017. p. 135-151.

14. Veness MJ. High-risk cutaneous squamous cell carcinoma of the head and neck. BioMed Res Int. 2007;2007:80572-80577.

15. International Commission on Radiation Units and Measurements (ICRU). Prescribing, Recording, and Reporting Photon Beam Therapy. ICRU Report 50. JICRU. 1993:26(1).

16. Khayat D, Rixe O, Martin G, et al. Surgical margins in cutaneous melanoma ( $2 \mathrm{~cm}$ versus $5 \mathrm{~cm}$ for lesions measuring less than $2.1-\mathrm{mm}$ thick) Long-term results of a large European multicentric phase III study. Cancer. 2003;97(8):1941-1946.

17. Cohn Cedermark G, Rutqvist LE, Andersson R, et al. Long term results of a randomized study by the Swedish Melanoma Study Group on $2 \mathrm{~cm}$ versus $5 \mathrm{~cm}$ resection margins for patients with cutaneous melanoma with a tumor thickness of 0.8-2.0 mm. Cancer.2000;89(7):1495-1501.

18. Moncrieff MD, Gyorki D, Saw R, et al. 1 Versus 2-cm excision margins for pT2-pT4 primary cutaneous melanoma (MelMarT): a feasibility study. Ann. Surg. Oncol. 2018;25(9):2541-2549.

19. Alieva M, van Rheenen J, Broekman ML. Potential impact of invasive surgical procedures on primary tumor growth and metastasis. Clin Exp Metastasis. 2018;35(4):319-331.

20. Gillgren P, Drzewiecki KT, Niin M, et al. 2-cm versus 4-cm surgical excision margins for primary cutaneous melanoma thicker than $2 \mathrm{~mm}$ : a randomised, multicentre trial. Lancet. 2011;378(9803):1635-1642.

21. Khan K, Mykula R, Kerstein R, et al. A 5-year follow-up study of 633 cutaneous SCC excisions: rates of local recurrence and lymph node metastasis. J Plast Reconstr Aes. 2018;71(8):1153-1158.

22. Chren MM, Torres JS, Stuart SE, et al. Recurrence after treatment of nonmelanoma skin cancer: a prospective cohort study. Arch Dermatol. 2011;147(5):540-546. 